\title{
MIRAR LA HISTORIA CON OTROS OJOS
}

\author{
MARÍA DE LOS ÁNGELES PÉrEZ SAMPER \\ GLORIA FRANCO RUBIO
}

Historia no hay más que una, pero existen muchas posibles miradas desde las que contemplarla. Con todas y cada una de las miradas de siempre y con algunas nuevas perspectivas como las que aquí proponemos, nuestro propósito es construir una historia más rica, más plena, una historia más compleja, pero más integrada. Se trata de superar las dicotomías, como las oposiciones entre idealismo y materialismo, entre sujeto y objeto, entre lo colectivo y lo individual. Todavía más, se trata de aprehender las realidades sociales como construcciones históricas y cotidianas de actores individuales y colectivos, construcciones que no siempre responden a la voluntad clara y al control de estos mismos actores. Frente al determinismo de las estructuras, hay que tener en cuenta, en la explicación de las prácticas, a los sujetos. No hay sociedad sin individuos y tampoco hay individuos sin sociedad. Y existe siempre un principio de incertidumbre.

En este camino, junto con otras teorías de la historia y de la sociedad, las orientaciones del sociólogo francés Pierre Bourdieu pueden resultar muy útiles para ayudar a construir una historia de la vida cotidiana, especialmente en la perspectiva que oscila entre sociabilidad y privacidad. Frente al subjetivismo voluntarista, los sujetos no actuarían libremente, sus prácticas estarían condicionadas por toda la historia anterior que habría sido incorporada en forma de habitus. El habitus es, según Bourdieu, una disposición, que se puede activar en diferentes conjuntos de relaciones y dar lugar a un abanico de prácticas distintas. El habitus se convierte así en principio no elegido de todas las «elecciones». La teoría de Bourdieu pretende sustituir la dicotomía entre individuo y sociedad en la explicación de las prácticas sociales, por la relación construida entre dos modos de existencia de lo social. Por un lado, las estructuras sociales externas, lo social hecho «campos» de posiciones sociales que se han construido en dinámicas históricas. Por otro, las estructuras sociales internalizadas, incorporadas al agente en forma de esquemas de percepción, pensamiento y acción: los habitus.

Fundamental es la tensión entre continuidad y cambio. La historia de la vida cotidiana es una historia de tiempos largos, de grandes resistencias, pero a la larga y en definitiva de evolución, de transformación, de cambio. El habitus tiende a asegurar su 
propia permanencia, es un mecanismo de defensa contra el cambio. Al incorporarse como esquema de percepción y apreciación de prácticas, operará una selección de las informaciones nuevas: «El peso particular de las experiencias primitivas resulta en efecto en lo esencial del hecho que el habitus tiende a asegurar su propia constancia y su propia defensa contra el cambio a través de la selección que opera entre las informaciones nuevas, rechazando, en caso de exposición fortuita o forzada, las informaciones capaces de poner en cuestión la información acumulada y sobre todo desfavoreciendo la exposición a tales informaciones. (...) Por la 'elección' sistemática que opera entre lugares, acontecimientos, personas susceptibles de ser frecuentados, el habitus tiende a ponerse al abrigo de las crisis y de las puestas en cuestión críticas asegurándose un medio al que está tan preadaptado como es posible.» ${ }^{1}$

Historia de la sociedad desde la perspectiva de lo cotidiano, pero siempre historia. Los conceptos de la sociología pueden contribuir al análisis, pero pretendemos hacer historia, por tanto trabajar desde la perspectiva del tiempo, que es el que hace la historia. Una historia que explique el pasado y que a la vez ayude a comprender el presente y a construir el futuro. Porque para explicar las prácticas de los agentes sociales, no basta con remitirlas a su situación presente. ${ }^{2}$ El concepto de habitus creado por Bourdieu reintrodujo la dimensión histórica en el análisis de la acción social, que asegura así la actuación del pasado en el presente: «Producto de la historia, el habitus produce prácticas (...) conformes a los esquemas engendrados por la historia; asegura la presencia activa de las experiencias pasadas que, depositadas en cada organismo bajo la forma de esquemas de percepción, de pensamiento y de acción, tienden, de forma más segura que todas las reglas formales y todas las normas explícitas, a garantizar la conformidad de las prácticas y su constancia en el tiempo.» Y añadía: «Historia incorporada, hecha naturaleza, y por ello olvidada en cuanto tal, el habitus es la presencia actuante de todo el pasado del que es el producto: de partida, es el que confiere a las prácticas su independencia relativa en relación a las determinaciones exteriores del presente inmediato. $»^{3}$ Hay que tener en cuenta siempre la importancia de la historicidad, pues el mundo social se construye a partir de lo ya construido en el pasado, las formas sociales del pasado son reproducidas, apropiadas, desplazadas y transformadas en las prácticas y las interacciones de la vida cotidiana de los actores y este trabajo cotidiano sobre la herencia del pasado abre un campo de posibilidades en el futuro. La temporalidad es esencial. Los sujetos han de producir sus prácticas en la urgencia temporal. Tienen que actuar aquí y ahora. No vale ningún modelo del sujeto que suponga una «reflexión» previa de todos los factores en juego -como la teoría del actor racional-. Pero tampoco vale una teoría que reduzca al sujeto a mero autómata, porque las respuestas han de darse en una enorme variedad de situaciones en las que siempre hay elementos distintos, nuevos.

1. Pierre Bourdieu: Le sens pratique, Paris Minuit, 1980, p. 102 (traducción española Taurus, 1992).

2. Pierre Bourdieu: Sociología y cultura, México, Grijalbo, 1990.

3. Pierre Bourdieu: Le sens pratique... pp. 91-94. 
A los tiempos hay que añadir los espacios, el privado y el público. El estudio de la «domesticidad», del ámbito doméstico, de la casa, del mobiliario y del ajuar que contiene, así como las prácticas de relación social que en ese ámbito se desarrollan, como pueden ser las representaciones teatrales, es primordial para la comprensión de la sociedad y de las redes de relaciones que la constituyen. Como señala Bourdieu: «El espacio habitado - y en primer lugar la casa- es el lugar privilegiado de la objetivación de los esquemas generadores y, por medio de las divisiones y de las jerarquías que establece entre las cosas, entre las personas y entre las prácticas, este sistema de clasificación hecho cosa inculca y refuerza continuamente los principios de la clasificación constitutiva del arbitrario cultural. (...) El mundo de los objetos, esta especie de libro donde toda cosa habla metafóricamente de todas las otras y en el que los niños aprenden a leer el mundo, se lee con todo el cuerpo, en y por los movimientos y los desplazamientos que hacen el espacio de los objetos tanto como son hechos por él. Las estructuras que contribuyen a la construcción del mundo de los objetos se construyen en la práctica de un mundo de objetos construidos según las mismas estructuras.» ${ }^{4}$

Otro factor fundamental en la teorización del habitus es su relación con el cuerpo. El habitus se aprende mediante el cuerpo -se incorpora-: mediante un proceso de familiarización práctica, que no pasa por la consciencia, con un universo de prácticas. Las maneras de la mesa pueden ser un buen ejemplo de esa incorporación. Como advierte Bourdieu: «No se terminaría de enumerar los valores hechos cuerpo, por la transubstanciación que opera la persuasión clandestina de una pedagogía implícita, capaz de inculcar toda una cosmología, una ética, una metafísica, una política, a través de órdenes tan insignificantes como 'ponte derecho' o 'no cojas tu cuchillo con la mano izquierda' y de inscribir en los detalles en apariencia más insignificantes del porte, de la postura o de los modales corporales y verbales los principios fundamentales del arbitrario cultural, situados así fuera del alcance de la consciencia y de la explicitación.» ${ }^{5}$

Las prácticas sociales no se explican, según Bourdieu, recurriendo a la consciencia de los actores, sino que suponen un sistema de relaciones que escapa a su aprehensión. Como los habitus se in-corporan, se aprenden con el cuerpo, más allá de la consciencia, y como suponen la interiorización de los esquemas cognitivos, perceptivos, apreciativos del grupo social en el que el sujeto es educado, se sigue que el sujeto reproduce estos esquemas, estos valores, de una manera involuntaria e inconsciente. Los sujetos están sujetados por los grupos sociales que los producen, por los esquemas que han incorporado: «Los esquemas del habitus, formas de clasificación originarias, deben su eficacia propia al hecho de que funcionan más allá de la conciencia y del discurso, luego fuera de las influencias del examen y del control voluntario: orientando prácticamente las prácticas, esconden lo que se denominaría injustamente unos valores en los gestos más automáticos o en las técnicas del cuerpo más insignificantes en apariencia (...) y ofrecen los principios más fundamentales de la construcción y de la

4. Pierre Bourdieu: Le sens pratique... pp. 129-130.

5. Pierre Bourdieu: Le sens pratique... p. 117. 
evaluación del mundo social, aquellos que expresan de la forma más directa la división del trabajo entre las clases, las clases de edad y los sexos, o la división del trabajo de dominación. ${ }^{6}$ Un sencillo paseo adquiere así trascendencia social, se integra en el entramado social y a la vez recrea la sociedad.

En las relaciones sociales se invierten y se intercambian capitales muy diversos. Bourdieu distinguía tres tipos de capital, el capital económico, integrado por dinero, propiedades, empresas. El capital social, formado por recursos basados en la pertenencia al grupo, las relaciones, las redes de influencia y apoyo, que definía como el conjunto de los recursos reales o potenciales, que están ligados a la posesión de una red duradera de relaciones, más o menos institucionalizadas, de conocimiento y reconocimiento mutuos. El capital cultural, que consiste en las formas de conocimiento, las habilidades, la educación, y las ventajas que tiene una persona, lo que les da un estatus más alto en la sociedad. Los padres proporcionarían a sus hijos el capital cultural mediante la transmisión de los valores, las actitudes y los conocimientos necesarios para tener éxito. El capital cultural puede adoptar diversas formas. El capital cultural interiorizado o incorporado, que es el que se adquiere en el seno de la familia o por medio de una circunstancia concreta, como pertenecer a una institución poderosa; el capital cultural objetivado, que es el visible en la acumulación de objetos extraordinarios, obras de arte que muestran el gusto distinguido; el capital cultural institucionalizado, cuya forma más evidente la constituyen los títulos y diplomas. Más tarde, Bourdieu añadiría otro tipo de capital, el capital simbólico, que consiste en los recursos disponibles para un individuo sobre la base del honor, el prestigio, el reconocimiento. Representativo de estos intercambios de los diversos tipos de capital puede ser el estudio del regalo y su significado, a la vez económico, social, cultural y simbólico.

La incorporación de los habitus está muy relacionada con otro tema central en la obra de Bourdieu: el «sentido práctico»o «racionalidad práctica». En este punto, la crítica es frontal a las teorías de la «racionalidad» que postulan un único modelo de racionalidad. Frente a un concepto abstracto y universal de «racionalidad» se impone la investigación concreta y específica de las diferentes «racionalidades prácticas» de los sujetos, que están en función de sus esquemas de acción y percepción -de sus habitus - formados en unas condiciones sociales y materiales particulares. ${ }^{7}$ No existe una opción única, sino opciones plurales, no existe una sola vía, sino múltiples itinerarios. Así una historia personal se inserta en el marco general histórico, pero no se pierde en él, sino que mantiene toda su singularidad. La vida de la princesa Pío puede ser un caso muy ilustrativo de esa «microhistoria» integrada en la gran historia.

Otra de las dimensiones fundamentales del habitus es su relación con las clases sociales y la reproducción social. El habitus se convierte así en una dimensión fundamental de la «clase social» de los sujetos: es la «clase incorporada»: a cada posición social distinta le corresponden distintos universos de experiencias, ámbitos de prácti-

6. Pierre Bourdieu: La distinción. Crítica social del gusto. Madrid, Taurus, 1988, p. 477.

7. Pierre Bourdieu: Raisons pratiques; sur la théorie de l'action, Paris. Seuil, 1994. 
cas, categorías de percepción y apreciación. Se distinguen, según Bourdieu, dos aspectos de la clase social: la clase objetivada-su posición en el sistema de relaciones sociales, sus condiciones materiales y culturales-y la clase incorporada -la clase social hecha cuerpo, habitus-. Este habitus de grupo será fundamental en la reproducción social. Porque, producido en unas determinadas condiciones sociales, y reproduciendo de manera corporal, inconsciente, los esquemas y divisiones de que es producto, funciona ajustado a las mismas condiciones, contribuyendo así a reproducirlas mediante su continua actualización. ${ }^{8}$

Todo ello se ve claramente en una de las dimensiones fundamentales del habitus: el sentido de los límites, de las posibilidades e imposibilidades. Por el habitus uno se incluye en aquello que considera propio y apropiado y por el habitus uno se excluye de lo que considera que está excluido. La idea de diferencia, de distinción, de desviación, fundamenta la noción misma de espacio, conjunto de posiciones distintas y coexistentes, externas unas a otras, definidas en relación unas de otras, por su exterioridad mutua y por relaciones de proximidad, de vecindad o de alejamiento y asimismo por relaciones de orden, verticales y horizontales. Y sobre todo se proyecta sobre las prácticas, comportamientos, conductas y actuaciones sociales, en su dimensión individual y sobre todo colectiva. Un elemento aparentemente tan sutil como el perfume se convierte así en un signo de distinción de gran significado social y cultural.

En la teoría de las prácticas de Pierre Bourdieu, estas deben explicarse como la relación entre dos sistemas de relaciones. Por un lado, el sistema de relaciones -históricamente construido- que constituye el «campo» específico en que se desarrolla la práctica. Por otro, el sistema de relaciones que produce a los agentes de las prácticas, su habitus, que nos remite a las condiciones sociales de la producción de estos agentes en su historia anterior. Sólo la puesta en relación de estos dos sistemas de relaciones permite eludir el objetivismo determinista y el subjetivismo voluntarista, planteando la «génesis» social de las estructuras de las prácticas desde una perspectiva relacional e histórica. Las realidades sociales son a la vez objetivadas e interiorizadas. Por una parte remiten a mundos objetivados, como reglas, normas, instituciones, exteriores a los agentes, que funcionan a la vez como condiciones limitantes y como puntos de apoyo para la acción, y por otra parte se inscriben en mundos subjetivos e interiorizados, constituidos principalmente por formas de sensibilidad, de percepción, de representación y de conocimiento.

Este número de la Revista de Historia Moderna. Anales de la Universidad de Alicante pretende ser una pequeña muestra de esas miradas plurales sobre la Historia, algunas de las muchas posibles. Sólo intenta sugerir las múltiples vías disponibles para iluminar una serie de perspectivas, que consideramos muy interesantes y reveladoras, pero hasta ahora poco conocidas y poco valoradas, y que pensamos que pueden contribuir a completar el panorama histórico e historiográfico.

8. P. Bourdieu y J.C. Passeron: La reproducción. Elementos para una teoría del sistema de enseñanza. Barcelona, Laia, 1977. 